\title{
Neutral dynamics with environmental noise: Age-size statistics and species lifetimes
}

\author{
David Kessler, ${ }^{1}$ Samir Suweis, ${ }^{2}$ Marco Formentin, ${ }^{3}$ and Nadav M. Shnerb ${ }^{1}$ \\ ${ }^{1}$ Department of Physics, Bar-Ilan University, Ramat-Gan 52900, Israel \\ ${ }^{2}$ Physics and Astronomy Department 'G. Galilei' \& CNISM, INFN, University of Padova, Via Marzolo 8, 35131 Padova, Italy \\ ${ }^{3}$ UTIA, Czech Academy of Sciences, Pod Vodárenskou věží 4, 18208 Prague, Czech Republic
}

(Received 15 March 2015; published 31 August 2015)

\begin{abstract}
Neutral dynamics, where taxa are assumed to be demographically equivalent and their abundance is governed solely by the stochasticity of the underlying birth-death process, has proved itself as an important minimal model that accounts for many empirical datasets in genetics and ecology. However, the restriction of the model to demographic $[\mathcal{O}(\sqrt{N})]$ noise yields relatively slow dynamics that appears to be in conflict with both short-term and long-term characteristics of the observed systems. Here we analyze two of these problems-age-size relationships and species extinction time-in the framework of a neutral theory with both demographic and environmental stochasticity. It turns out that environmentally induced variations of the demographic rates control the long-term dynamics and modify dramatically the predictions of the neutral theory with demographic noise only, yielding much better agreement with empirical data. We consider two prototypes of "zero mean" environmental noise, one which is balanced with regard to the arithmetic abundance, another balanced in the logarithmic (fitness) space, study their species lifetime statistics, and discuss their relevance to realistic models of community dynamics.
\end{abstract}

DOI: 10.1103/PhysRevE.92.022722

PACS number(s): 87.10.Mn, 87.23.Cc, 64.60.Ht, 05.40.Ca

\section{INTRODUCTION}

The theory of population and community dynamics is a central mathematical tool in many branches of life science, including genetics, ecology, and evolution. This broad field of research is dominated by two competing and complementary paradigms. Darwinian natural selection suggests that the fitness of phenotypes and species is different and stresses deterministic effects like the survival of the fittest, downplaying the role of noise and fluctuations. On the other hand, neutral theories assume that selective effects are relatively weak and different taxa (or haplotype etc,) admit almost identical fitness, so that the main driver of population dynamics is stochasticity.

Within the neutral framework, first Kimura's theory of molecular evolution [1] and more recently Hubbell's universal neutral theory of biodiversity (UNTB) [2-4] have both attracted a lot of attention. In the latter case, the successful explanation of empirical species abundance distribution curves by a simple theory with only two parameters [5,6] appears as a very appealing minimalistic model, especially when compared to niche-based approaches that usually require the reconstruction of many parameters (such as the relative fitness of species), a very difficult task in high-diversity assemblages [7].

These neutral models assume that the main driver of community dynamics is demographic stochasticity, i.e., the noise embodied in the birth-death process of individual agents, with (if the whole community has to keep a fixed size) the expected number of descendants for each individual being precisely 1 . Accordingly, a population of $N$ individuals will produce $N$ offspring on average, and the per-generation fluctuations will be proportional to $\sqrt{N}$, which is quite a weak noise in the limit of large $N$. This restriction of the neutral model to pure demographic noise leads to a few severe difficulties when its predictions are compared with empirical patterns.

On evolutionary time scales, the two main unsolved problems are the age-size relationships and species extinction time. In a neutral theory with pure demographic noise both the age (measured in generations) of a species and its time to extinction are proportional to its abundance. This time scale is ridiculously long for, inter alia, various species of trees (with generation time of $50 \mathrm{y}$ ) and for passerine birds (generation time $3 \mathrm{y}$ ), as noted by many authors [8-11]. The fossil data, which indicate that species lifetime is typically a few million years, is again in contradiction to the $N$ generation estimate for common species. As Robert Ricklefs summarized his findings [9], "drift is simply too slow to account for the rate of turnover of passerine birds." The idea of protracted speciation [12], suggested to account for the apparent underrepresentation of rare species and their relatively long lifetime, cannot resolve these difficulties.

On the ecological time scale, empirically observed fluctuations in abundance are usually too strong to be explained by UNTB [13-16]. Moreover, UNTB cannot explain the scaling of fluctuations variance with population size: with pure demographic noise the theory predicts a linear scaling but empirical analyses show a prevalence of superlinear dependence [17]. The decay of community compositional similarity is again much faster than the UNTB predictions [18].

A very plausible (and one in any case necessitated by biological reality) generalization of the neutral theory that may resolve many of these problems while preserving the minimalistic character of the model is to add environmental stochasticity to the dynamics. In nature the demographic response of individuals to environmental variations is (at least partially) correlated within species, while different species may show transient fitness advantage at various times due to differences in their temporal niches. In our proposed generalized neutral model all individuals are demographically equal on average but the relative fitness of a population fluctuates in time. Environmental stochasticity generates $\mathcal{O}(N)$ short-term fluctuations in population abundance, closer in size to those observed in reality.

To provide an order of magnitude estimate for the strength of environmental noise in empirical systems, the variance through time plot of the logarithmic abundance 

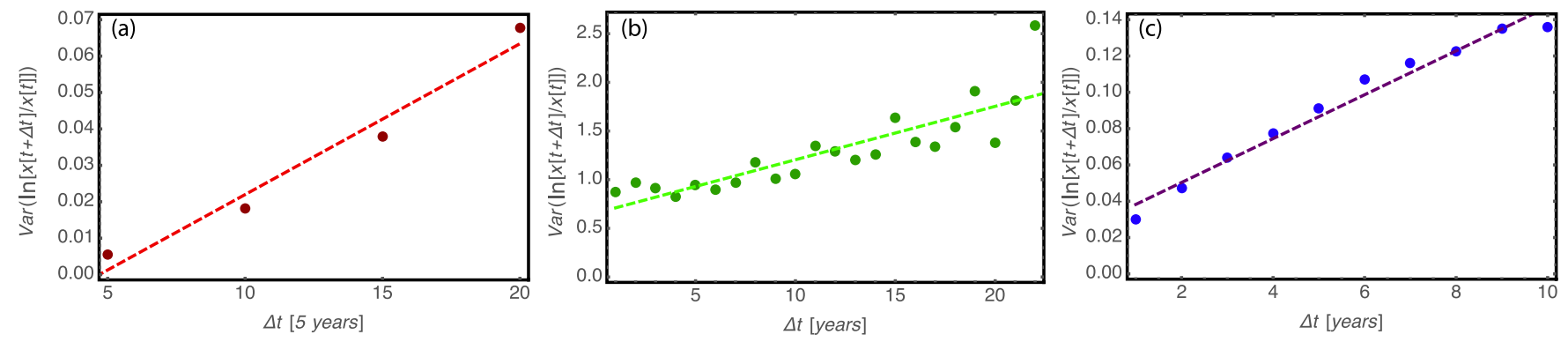

FIG. 1. (Color online) Variance through time plots for three empirical datasets: (a) Trees of the tropical forest in Barro Colorado Island (BCI), considering trees with diameter $>10 \mathrm{~mm}$ (data are from www.ctfs.si.edu) from 1990 to 2005 (every 5 y). (b) Fish samples collected from the cooling-water filter screens at Hinkley Point B Power Station in Bristol, from 1980 to 2008 [19]. We only consider estuarine fish species (not crustaceous organisms). (c) Herbaceous plant dataset [20] comprising a series of 51 quadrats of $1 \mathrm{~m}^{2}$ from mixed Kansas grass prairies where all individual plants were mapped every year from 1932 to 1972 . In all cases we gathered $\ln \left(N_{t+\Delta t} / N_{t}\right)$ (natural logarithm was used) from every two same species abundance observations that are $\Delta t$ apart, and plotted the variance of this quantity vs $\Delta t$ (the details of this technique are presented in Ref. [15]). $m$ denotes the angular coefficient of the linear fit of data points $\left[\mathrm{m}=4 \times 10^{-3}\right.$ for (a), $5.5 \times 10^{-2}$ for (b), and 0.01 for (c)], while $P_{v a l}\left[0.01\right.$ for (a), $P<10^{-4}$ for (b) and (c)] and $R^{2}$ [0.97, 0.72 and 0.97 for (a), (b), and (c) correspondingly] give the significance and the goodness of the fit, respectively. In all three cases the variance appears to grow linearly in time, as suggested for a system with logarithmically balanced environmental noise, where the slope $m$ indicates the value of the effective $D$ defined in Eq. (3). The intercept of the linear fit is above zero for the fish and the grassland, indicating the effect of sampling errors [15]. The negative intercept for trees indicates a delayed response of the system to the changing environment. Since the time scales are relatively small, the same analysis for arithmetically balanced noise, where the variance of $\left(N_{t+\Delta t}-N_{t}\right) / N_{t}$ is plotted against $t$, yields almost the same results (not shown).

ratio is presented in Fig. 1 for three empirical datasets, showing the linear increase characteristic of environmental stochasticity. As discussed in Ref. [15], the slope of this curve is proportional to the strength of environmental variations. In the discussion section we plug this number into our mathematical expressions to show that realistic noise indeed solves the UNTB time-scale problem.

Our first major result, then, is that a neutral model with both demographic and environmental noise may solve the mismatch between the predictions of the purely demographic UNTB and the empirical evidence, shortening significantly the number of generations needed for the ancestry of a single founder to reach large abundance. The linear scaling of time (in generations) with the abundance $N$ is replaced by logarithmic scaling, yielding reasonable age-size estimations. Our second result is that the time to extinction of an abundant species is shortened significantly. As described below, the various possibilities obtained for species lifetime statistics correspond to previous, evidence-based suggestions.

The implementation of environmental stochasticity into a neutral model poses an important conceptual problem. As noticed a while ago [21] there are two different scenarios for balanced (zero mean) environmental stochasticity: the balance may be either arithmetic (i.e., the noise statistics is such that the mean abundance is kept fixed; for example if during a bad year the population shrinks by $1 / 2$, during a good year it grows by $50 \%$ ) or logarithmic (in a bad year it shrinks to $1 / 2$, say, and in a good year the population doubles). These two scenarios are analogous to the use of Ito or Stratonovich calculus in the white noise limit [22,23].

For a fixed-size community, where different species are playing, more or less, a zero sum game, none of these scenarios provide a satisfactory description of the dynamics. Under arithmetically balanced noise all species eventually go extinct with probability 1 , while under log-balanced noise the size of the community grows exponentially, both in contrast with the fixed size requirement. Introducing environmental stochasticity into the UNTB is a delicate task, in which the interplay between environmental and demographic fluctuations and other effects should be taken into account. Here we do not solve this problem, although we provide some preliminary considerations in the discussion section.

However, any model with balanced noise must be somewhere between the log-balanced and the arithmetic-balanced extremes considered here and, as will be shown below, in both cases the abundance-age relationships are logarithmic so the species lifetime problem is solved in any case. On the other hand, the log and the arithmetic dynamics differ dramatically with respect to species lifetime: the $t^{-2}$ tail of lifetime statistics predicted by the purely demographic theory [24] is replaced by a slower $\left(t^{-3 / 2}\right)$ decay if the noise is $\log$ balanced and by much faster exponential decay for arithmetically balanced stochasticity. In the literature one can find empirical evidence for all these behaviors [25,26]; the combination of demographic and environmental stochasticity is endowed with the required flexibility to account, in the appropriate regime of parameters, for many observed patterns of species and genus lifetime statistics.

\section{THEORETICAL FRAMEWORK}

Demographic noise reflects variability in reproductive success which is uncorrelated among individuals in the population. This noise is fully characterized by $P_{n}$, the probability of an individual to produce $n$ offspring during its lifetime. The average number of offspring is $\sum_{n} n P_{n}=R_{o}$, and for a fixed size population $R_{o}=1$. Another important parameter is the variance of the number of offspring $\sigma^{2}$, which measures the differences in reproductive success among individuals: in animal and human populations, where the number of offspring is between 0 and 10 , say, and $P_{n}$ decays sharply with $n$, the variance is of order unity; for a virus population, where some 
viruses infect a cell and produce 10000 offspring while the others die childless, the variance is much larger.

The theory of a fixed-size population under pure demographic noise traces back to the work of Galton and Watson [27]. In Appendix A we present a generating function analysis of this case. Species lifetime statistics are reflected in $\delta(t)$, the chance of the lineage of a single individual to survive until $t$ (time is measured in units of generations), which is shown to satisfy

$$
\frac{d \delta}{d t} \sim-\frac{\sigma^{2}}{2} \delta^{2}(t) .
$$

This implies that the survival probability of a species decays at long times like $1 / t$ and that the statistics of species lifetime admits a $1 / t^{2}$ tail [24]. Since the average abundance of a species is constant, the fact that only a fraction $2 /\left(\sigma^{2} t\right)$ of the species survive by time $t$ implies that their mean abundance should grow linearly with $t$,

$$
N_{\text {surv }}(t) \sim \frac{\sigma^{2}}{2} t,
$$

where $N_{\text {surv }}(t)$ is the average population abundance at $t$, conditioned on survival. These results demonstrate the two main problems of a neutral theory with pure demographic noise, when confronted with empirical findings and a priori considerations: First, the time needed for a species to reach abundance $N$ (starting with one individual, i.e., point speciation) is typically of order $N$ generations. Second, the typical time to extinction of a species of abundance $N$ is again of order $N$ generations, since the theory is neutral and the ancestry of any individual evolves independently (formally the average time to extinction diverges due to the $1 / t^{2}$ tail). Slight modification of the model, like implementing a zero-sum game in the community (such that the size of the community is kept fixed in the strong sense, as opposed to keeping the average size fixed by assuming $R_{o}=1$ ), does not significantly change these conclusions [11].

Adding environmental stochasticity to this model implies that, as the environmental conditions vary, the demographic success of the whole population varies accordingly, so the average reproductive success $R_{o}$ becomes time dependent. In some cases, discussed in Ref. [18], the addition of environmental noise leads to a stabilization of the populations around some equilibrium value because of the (quite counterintuitive) storage effect. This paper deals with models that have no such effect, a set which includes any environmental noise generalization of the dynamics considered in Ref. [5].

Environmental conditions admit some correlation time $T$. Speaking about a good or bad year (in terms of precipitation, winds, etc.) one assumes that the environment (in the general sense, including the effects of competition with other species) was, in general, favorable or hostile to a specific species during this period. If the demographic rates are kept fixed during $T$ and the abundance of a certain species at time $s$ is $N_{s}$, then typically $N_{s+1}=e^{\gamma_{s}} N_{s}$ where $\gamma_{s}=\left(R_{o}-1\right) T$. The simplest way to define a "balanced" environmental noise is to assume that $\gamma_{s}$ (the fitness parameter, or the deviation of $R_{o}$ from unity) is an identically distributed random variable with zero mean and variance,

$$
\overline{\gamma_{s}^{2}} \equiv 2 D
$$

where the overbar denotes an average. In this scenario the steps are balanced in the logarithmic space [the expectation value of $\ln (N)$ is kept fixed] but the arithmetic mean of $N$ is growing in time, since $\overline{\exp (\gamma)}>1$. The reason for this is the asymmetry between growth and decline: since the per capita growth (or decay) rate is kept fixed for some time, the overall demographic benefit for the population includes not only the birth originated from the individuals that were present at $s$, but also from the individuals that were born between $s$ and $s+1$, and the opposite is true in the case of decline. The response of the population to varying environmental conditions has an "inertia" that increases the overall demographic benefit during good times relative to the loss suffered during bad times, hence producing a net bias towards growth.

However, in many scenarios the per-capita growth rate decreases when the population increases. For example, in a fixed size community with a zero sum game like the one considered in Ref. [28], the fitness of a species determines its chance to replace an individual of another species by its own offspring; the more abundant a species is, the greater the chance of intraspecific competition so the inertia is weaker. In its extreme limit one can model this kind of behavior by taking $N_{s+1}=\gamma_{s} N_{s}$, where $\gamma_{s}$ is again a balanced noise. Now it is the expectation value of $N$ [rather than $\ln (N)]$ which is kept fixed. We define these two types of environmental noise as logarithmically balanced (case A, where the "opposite" of $N \rightarrow N / 2$ is $N \rightarrow 2 N$ ) and arithmetically balanced (case B, the "opposite" of $N \rightarrow N / 2$ is $N \rightarrow 3 N / 2$ ). Realistic systems with environmental stochasticity are, most likely, somewhere between these two extremes, but the solutions we present below for these two scenarios provide the basic insights needed for consideration of the generic case, as explained in the discussion section.

\section{AGE-ABUNDANCE RELATIONSHIPS AND LIFETIME STATISTICS}

Within the above theoretical framework, we have developed and solved a model of population dynamics under both environmental and demographic noise, making a distinction between logarithmically balanced (case A) and arithmetically balanced (case B) stochasticity. The results have been derived for a model with a geometric distribution of offspring, but, as explained in Appendix C, they are valid for any realization of the demographic noise provided that one is interested in the long-term behavior of the system.

\section{A. Survival probability}

First we consider the survival probability, assuming a single individual (point speciation) at $t=0$. It turns out (see Appendixes $B$ and $C$ ) that the survival probability at long times is governed by the equations

$$
\begin{aligned}
& \frac{d \delta(t)}{d t}=\gamma_{t} \delta(t)+D \delta(t)-\frac{\sigma^{2}}{2} \delta^{2}(t), \quad \text { case A, } \\
& \frac{d \delta(t)}{d t}=\gamma_{t} \delta(t)-\frac{\sigma^{2}}{2} \delta^{2}(t), \quad \text { case B. }
\end{aligned}
$$

In the limit $\gamma=D=0$ both equations reduce to (1), the equation obtained previously for pure demographic noise, as 

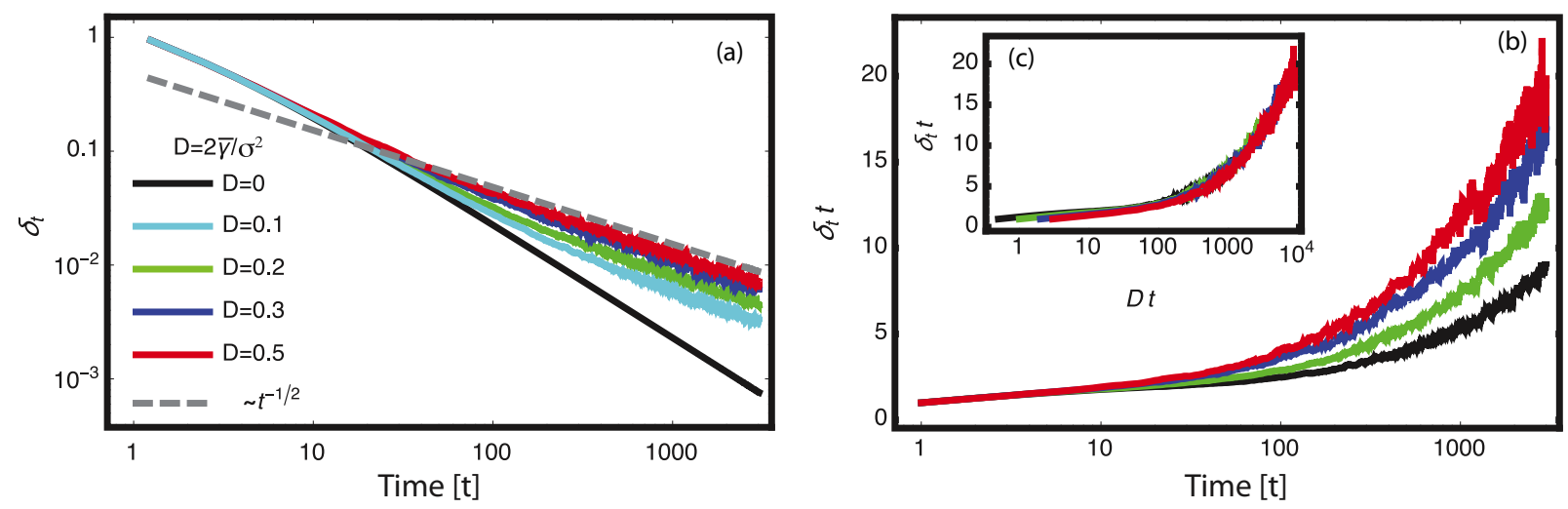

FIG. 2. (Color online) (a) A plot of $\delta_{t}$, the chance to survive at time $t$ for a certain levels of overall diffusion coefficient $D$ [log-log scale, the lines correspond to different $D$ values in the legends are ordered from bottom (low) to top (high $D$ ) in both panels]. The simulated process is the standard Wright-Fisher process with nonoverlapping generations. (b) Plotting $\delta_{t} t$ vs time in linear-log scale. (c) A plot of $\delta_{t} t$ against $t D$. In this case the data collapse as predicted by Eq. (B7). This collapse suggests that the proper scaling of the cumulative survival probability is $\delta_{t}=\frac{\mathcal{F}(t D)}{t}$, with $\mathcal{F}(x) \sim x^{1 / 2}$ for $x \rightarrow \infty$ and $\mathcal{F}(x) \sim$ const when $x \rightarrow 0$.

expected. Moreover, substituting $\delta=2 D \tilde{\delta} / \sigma^{2}$, changing the time units with $t=\tau / D$, and rescaling the noise term to unit white noise, one finds a $D$ independent equation. This implies that a plot of $\delta t \sim \tilde{\delta} \tau$ vs $D t=\tau$ should be independent of the strength of the environmental noise.

From Eq. (B7) one may extract the long-time asymptotics of the survival probability. When the environmental stochasticity is relatively weak the short-time chance of extinction is controlled by the demographic noise. This leads us to propose that, for both the logarithmic and the arithmetic case,

$$
\delta(t)=\frac{\mathcal{F}(t D)}{t},
$$

where the form of $\mathcal{F}(t D)$ is obtained analytically in Appendix B 2.

In case $\mathrm{A}(\log$ balanced) $\mathcal{F}(x)$ approaches $\sqrt{x / \pi}$ when $x \rightarrow \infty$ and unity when $x \rightarrow 0$. Accordingly, the chance of extinction at the $t$ th generation crosses over from the GaltonWatson universal limit (characterizing a process with pure demographic noise) $t^{-2}$ to the first passage time asymptotics $t^{-3 / 2}$ at $t^{*} \sim 1 / D$.

The intuition behind this result is clear. In the absence of demographic noise, the population performs an unbiased random walk in the logarithmic space, hence it will survive until it reaches a threshold at, say, $\ln (N)=0$ (a single individual). The theory of first-passage time for a $1 d$ random walker tells us that at long time the chance to survive decays like $t^{-1 / 2}$. Since populations that stay alive for a long time typically reach high abundance, the long-time behavior is controlled by this term [29], while at shorter time scales environmental stochasticity is too weak and the behavior is controlled by the $t^{-1}$ term of the purely demographic process.

Figure 2 shows simulation results for the survival probability in case A, i.e., the chance that the system did not go extinct until $t, \delta(t)$. Indeed, this quantity decays like $t^{-1}$ when the process is purely demographic, and it switches to $t^{-1 / 2}$ behavior at long times when the system is subject to environmental noise. Moreover, when $t \delta(t)$ is plotted against $D t$ the data collapse as predicted above.
In case $\mathrm{B}$ (arithmetic balance) $\mathcal{F}(x)$ approaches $\exp (-x / 4)$ when $x \rightarrow \infty$ and unity when $x \rightarrow 0$, as demonstrated in Fig. 3. Again the short-term behavior is controlled by demographic noise, but the long-term survival probability is no longer a power law. The reason is that, unlike the log-balanced noise, in case B most of the species are shrinking in time (as can be seen easily by tracing the $1 / 2-3 / 2$ and $1 / 2-2$ processes to the next generations).

Returning to the empirical species lifetime problem, for a neutral theory with pure demographic noise the number of generations needed for a population to shrink to zero is typically its abundance, yielding unrealistic lifetimes for common species. When environmental noise is introduced, the long-term dynamics (where long is relative to the strength of the environmental noise, i.e., $t \sim 1 / D$ ) is dominated by $x=$ $D t$, so a decent amount of environmental stochasticity will shorten significantly the lifetime and will solve the problem

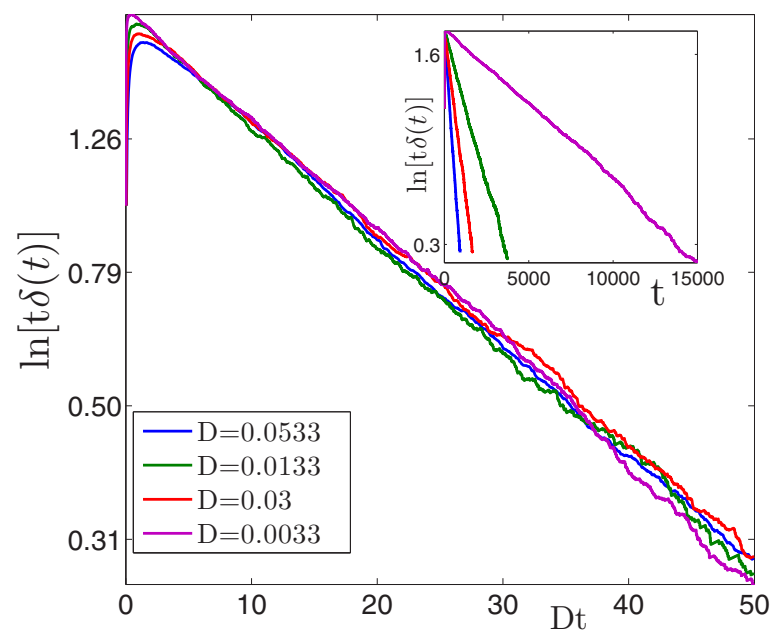

FIG. 3. (Color online) The survival probability of a species $\delta$ vs time in case B (arithmetically balanced noise). The logarithm of $t \delta(t)$ is plotted against $t$ (inset), showing an exponential decay with $D$ dependent slope. When plotted against $D t$ (main panel) the data collapse, indicating the dependence of $\mathcal{F}$ on $D t$. 
of $\mathcal{O}(N)$ generations scaling. Regarding the statistics of the species lifetime distribution, all the possibilities considered here-scaling with $t^{-3 / 2}$ in case $\mathrm{A}, t^{-2}$ for pure demographic noise and exponential decay-have been suggested in the literature based on the analysis of fossil data; see [24] and references therein.

\section{B. Age-abundance relationships}

Now let us turn to the dependence of abundance on the species' age. In Appendix B we define and calculate $\bar{\zeta}(t)$, the mean abundance at time $t$ of the ancestry originated from a single individual at $t=0$. However, when examining an empirical community one considers only the species that have not yet gone extinct, so the relevant quantity for the age-abundance relationships is the average size of a species at $t$ conditioned on nonextinction, $N_{\text {surv }}(t)=\zeta(t) / \delta(t)$. As shown in Appendix B 3, there are strong differences between logarithmic and arithmetic noise, although the bottom line is similar $t^{-1 / 2}$

In case A (log balanced) $\zeta(t) \sim \frac{2}{D}\left(e^{D t / 2}-1\right)$. Since $\delta \sim$

$$
N_{\text {surv }}(t) \sim \frac{2}{D t^{1 / 2}} e^{D t / 2} .
$$

Thus the abundance of the surviving species, instead of growing linearly, is growing exponentially in time. In Appendix B 4 we explain that the average result presented here and the typical result differ from each other due to the skewness of the distribution, so in the typical case the growth of $N_{\text {surv }}$ is subexponential,

$$
N_{\text {surv }}^{\mathrm{typ}}(t) \sim e^{\sqrt{2 D t}} \text {. }
$$

For case $\mathrm{B}$, on the other hand, $\zeta=1$, since the noise is balanced in the real space. On the other hand $\delta$ is decaying exponentially, so the net result is, again,

$$
N_{\text {surv }}(t) \sim e^{D t / 4} \text {. }
$$

\section{DISCUSSION AND CONCLUSIONS}

The failure of the UNTB to account for dynamic patterns of populations and communities has been known for a long time [13], and was stressed recently by many authors. Basically, the $\mathcal{O} \sqrt{N}$ scaling of the demographic noise makes it inadequate to account for the observed fluctuations on all time scales. The tempo of the dynamics may be accelerated if one assumes a very large value of $\sigma^{2}$ (as suggested, essentially, in Ref. [10]; see [11]) or by keeping the generation time as a free parameter (see, e.g., [30]), but any of these approaches carries its own difficulties.

On the other hand, environmental stochasticity is known to be ubiquitous in living systems, affecting communities even under the most stable conditions (see, e.g., [31]). Incorporating this mechanism into the neutral theory is a required step in any case [32]. We have already showed that environmental noise increases substantially the heterogeneity of the species abundance distribution [see [28], Eq. (3)], a feature that may account for the empirical results analyzed in Ref. [7]. The fact that this noise increases temporal fluctuations and decreases the time scale makes this project even more attractive.
Under environmental stochasticity, the demographic rates of all individuals belonging to a species (roughly speaking, their fitness) are fluctuating coherently in time, and the species abundance varies accordingly. As explained, the environmental noise may be "neutral" in two different senses. One scenario is when the relative fitness, when averaged over time, will be zero, this corresponds to logarithmically balanced noise or case A considered above. The other scenario, case $\mathrm{B}$, occurs when the time average of the demographic gain is zero.

As shown above, these two cases correspond to two different species lifetime statistics. The chance of a species to survive decays like $1 / t^{3 / 2}$ in case $\mathrm{A}$ and exponentially in case B. All these possibilities, $-3 / 2$ law, -2 law, and exponential, were suggested for the tails of species lifetime distributions as extracted from fossil data [24]. More important is the transition of the general scaling from $t$ (measured in units of generations) to $D t$, allowing the environmental noise to control the extinction times.

For the average abundance of a species at time $t$ after point speciation conditioned on nonextinction, $N_{\text {surv }}(t)$, we obtained exponential growth (in case B) and a typical stretched exponential growth (in case A). This appears to solve the "agesize" problem [8,9]: while the time to the most recent common ancestor scales with abundance in a purely demographic neutral model, it scales with the logarithm (or logarithm squared) of $N$ in the presence of environmental stochasticity. Equations (7) and (8) suggest that the time from speciation to abundance $N$ scales like $\ln (N) / D$ generations (case B) or with $\ln ^{2} N /(2 D)$ (case A). If, for example, one considers a set of $10^{9}$ conspecific trees for a frequent species in the Amazon basin (this is close to the contemporary figure; see recent survey in Ref. [33]), with about a 50-y generation time, the neutral theory suggests $50 \times 10^{9}$ years, more than the age of the universe. On the other hand, the left panel of Fig. 1 suggests that, measured in units of a single generation (50 y) $D \sim 0.1$, so the neutral theory with environmental noise modifies the speciation time to about 50000 years before present (case B) or about $150000 \mathrm{ybp}$ (case A). This, of course, is an extrapolation since the environmental noise may be either much smaller (if some of the short-term fluctuations are averaged out due to balancing effects like the increase of species specific parasites) or much larger (if extreme events do not appear in the half-generation window considered), but the scales are clearly small enough to solve the age-abundance problem even if the estimation for $D$ is modified by an order of magnitude.

Finally, we would like to comment about the generic case. When a community is subject to a stabilizing mechanism that keeps its size fixed or almost fixed, the dynamics of a single species must be somewhere between the two extremes considered in this paper. Pure case A dynamics cannot hold as the average population increases exponentially, while pure $\mathrm{B}$ dynamics is rejected since all species go extinct with probability 1 so the size of the community must shrink eventually to 0 . In Fig. 4 we plot the mean of $\left(N_{t+\Delta t}-N_{t}\right) / N_{t}$ (positive in pure case $\mathrm{A}$, vanishes in case B) and the mean $\ln \left(N_{t+\Delta t} / N_{t}\right)$ (vanishes in pure case A, negative in case B) vs $N$ as extracted from simulation of a community model with environmental noise (species specific randomly varying 


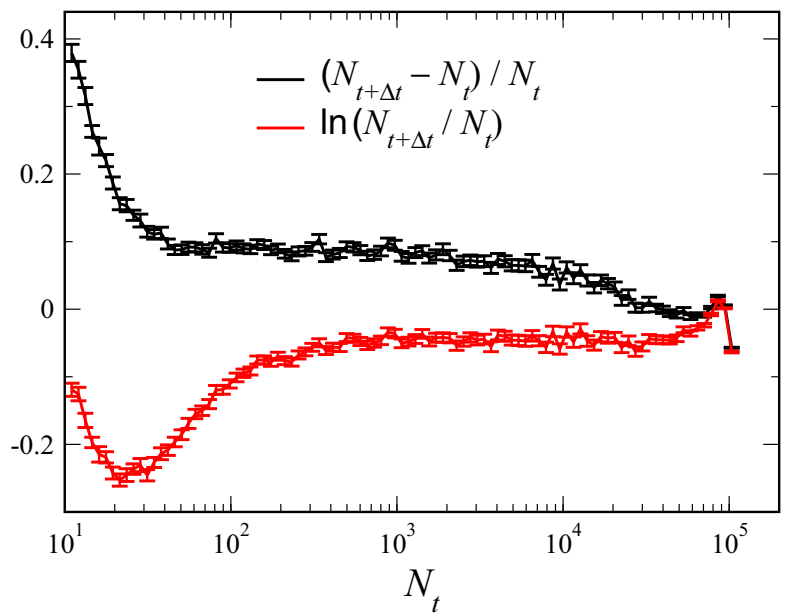

FIG. 4. (Color online) The mean of the logarithmic (red) and arithmetic (black) abundance fluctuations as a function of the abundance, extracted from simulation of a neutral community model with both demographic and environmental noise. A Moran-type continuous time process with discrete individuals was simulated. At each small time step $(d t=0.1)$ we generate the number of births and deaths for each species. The number of births is a Poisson distributed number with mean $\alpha_{i}(t) d t$, where $\alpha_{i}(t)$ is the instantaneous growth rate (fitness) of species $i$. The number of deaths in the species is binomially distributed, with the probability $N_{\text {tot }} / K$ per individual, where $N_{\text {tot }}$ is the instantaneous size of the community and $K=10^{5}$. In addition, a Poisson number of new immigrants is drawn, with mean $\mu d t$, where $\mu=2$. Each immigrant founds a new species. The birth rates $\alpha_{i}$ are given by $\alpha_{i}=\exp \left(\gamma_{i}\right)$ where the $\gamma_{i}$ are generated by an Orenstein-Uhlenbeck process, $\dot{\gamma}_{i}=-\gamma_{i} / \tau+\eta$, so that they are Gaussian distributed with mean 0 and variance 0.001 and correlation time 2 . This way the birth rates are guaranteed positive.

fitness). The situation is clearly between case A and case $\mathrm{B}$ - the arithmetic mean is slightly positive, the log mean is slightly negative - and shows some nontrivial $N$ dependence.

Our approach shows how a rich behavior may arise in a simple model where we do not model interaction among different species. Further works will investigate the effect of environmental noise when considering a whole ecological community where species explicitly interact among each other.

\section{ACKNOWLEDGMENTS}

D.A.K. acknowledges the support of the Israel Science Foundation. N.M.S. acknowledges the support of Israel Science Foundation BIKURA Grant No. 1026/11. S.S. thanks the Physics \& Astronomy Department, University of Padova, for financial support through Senior Grant No. 13.32.2. M.F. has been partially supported by Grantová agentura Ceské republiky Grant P201/12/2613. .

\section{APPENDIX A: SURVIVAL PROBABILITY IN A MODEL WITH PURE DEMOGRAPHIC NOISE}

We start by considering an event of point speciation, i.e., a new taxon that appears as one individual, the founder. Let us denote the chance that a single individual has $n$ descendants in the $s$ th generation by $P_{n}^{(s)}$. In a constant population, perfectly neutral scenario with pure demographic noise, the number of offspring $n$ that every given individual produces is independent of time and identically distributed, given by $P_{n}^{(1)}$. The average number of offspring is unity, i.e., $\sum_{n} n P_{n}^{(1)}=1$ and the strength of the demographic noise is characterized by $\sigma^{2} \equiv \sum_{n} n(n-1) P_{n}^{(1)}$. This scenario was analyzed by Galton and Watson $(\mathrm{GW})$, who showed that such a population goes extinct with probability 1 [27].

To generalize the GW result, one defines the generating function

$$
G^{(1)}(x)=\sum_{n} x^{n} P_{n}^{(1)}
$$

and its generalization to $s$ generations. For example, $P_{n}^{(2)}$ is the chance to have $n$ grandchildren, while $G^{(2)}(x)$ is the corresponding generating function. The successive generating functions are given by

$$
G^{(s)}(x)=G^{(1)}\left[G^{(s-1)}(x)\right] .
$$

By definition, $G^{(s)}(0)=P_{0}^{(s)}$ is the probability that the lineage originating from the founder went extinct by the $s$ th generation, and $G^{(s+1)}(0)-G^{(s)}(0) \approx d G^{(s)}(0) / d s$ determines the chance of extinction at the $s$ generation. After many generations the chance of extinction is almost 1 , so $G^{(s)}(0)=1-\delta_{s}, \delta_{s} \ll 1$. Equation (A1) implies that

$$
\begin{aligned}
1-\delta_{s+1} & =\sum_{n} P_{n}^{(1)}\left(1-\delta_{s}\right)^{n} \\
& \approx \sum_{n} P_{n}^{(1)}\left[1-n \delta_{s}+n(n-1) \delta_{s}^{2} / 2\right],
\end{aligned}
$$

which leads to the recursive equation

$$
\delta_{s+1}=\delta_{s}-\sigma^{2} \delta_{s}^{2} / 2 .
$$

Taking the continuum limit we then obtain that the survival probability is determined by the differential equation

$$
\dot{\delta}(t) \sim-\sigma^{2} \delta^{2}(t) / 2 .
$$

This result implies that the long-time decay of the survival probability goes like $\delta(t) \approx 2 /\left(\sigma^{2} t\right)$, so the statistics of species lifetime admits a $1 / t^{2}$ tail [24].

\section{APPENDIX B: SURVIVAL PROBABILITY AND AGE-ABUNDANCE RELATIONSHIPS IN A MODEL WITH BOTH DEMOGRAPHIC AND ENVIRONMENTAL NOISE}

\section{Neutral dynamics with environmental noise: The geometric neutral process}

In this section we derive our main results, implementing a model with a geometric distribution of offspring. As explained in the main text, two cases are considered here: a logarithmically balanced noise and an arithmetically balanced noise.

In a neutral model with a geometric distribution but without any stochasticity, the chance of an individual to produce $n$ offspring is $P_{n}=1 / 2^{n+1}$ and the corresponding generating 
function for a single generation is

$$
G^{(1)}(x)=\sum_{n} x^{n} P_{n}=\frac{1}{2-x} .
$$

What makes this model easy to handle is the convenient structure of the generating function. The generating function for the population after $s$ generations is obtained by iterating $G^{(1)}$, and in this specific case the answer is immediate [34],

$$
\begin{aligned}
G^{(s)}(x) & \equiv \sum_{n=0}^{\infty} x^{n} P_{n}^{(s)}=G(G(G \ldots \text { s times }(G(x)))) \\
& =\frac{s-(s-1) x}{(s+1)-s x} .
\end{aligned}
$$

Thus the distribution, apart from $P_{0}$, remains geometric. From this one can easily derive the results given above for a purely demographic model for this special case of geometrically distributed births.

To construct a model combining both demographic and environmental noise, we consider a discrete time dynamics, where for convenience we choose the time step to equal the generation time, so that all individuals of the $s$ th generation reproduce and then simultaneously pass from the scene.

Now let us consider the two random processes defined in the main text. The probability for each individual at the $s$ th generation to produce $n$ offspring conditioned on an environmental noise determined $\gamma_{s}$ is

$$
\begin{aligned}
& P\left(n \mid \gamma_{s}\right)=\frac{e^{\gamma_{s} n}}{\left(e^{\gamma_{s}}+1\right)^{n+1}}, \quad \text { case A, } \\
& P\left(n \mid \gamma_{s}\right)=\frac{\left(1+\gamma_{s}\right)^{n}}{\left(2+\gamma_{s}\right)^{n+1}}, \quad \text { case B, }
\end{aligned}
$$

where case $\mathrm{A}$ is the logarithmically balanced noise and case $\mathrm{B}$ corresponds to arithmetically balanced noise.

When $\gamma_{s}=0$ for any $s$ one obtains, of course, a purely demographic process. For nonzero $\gamma_{s}$ the fitness (or the deterministic growth rate) of the population fluctuates, and $D \equiv \overline{\gamma^{2}} / 2$ characterizes the strength of the environmental noise. The model is neutral in the sense that $\gamma_{s}$ is distributed identically for all species and so considered over long time scales, all species are demographically equivalent.

The generating functions in the two cases are then

$$
\begin{aligned}
G^{(1)}\left(x \mid \gamma_{s}\right) & =\frac{1}{1+e^{\gamma_{s}}-x e^{\gamma_{s}}}, \quad \text { case A, } \\
G^{(1)}\left(x \mid \gamma_{s}\right) & =\frac{1}{2+\gamma-x(1+\gamma)}, \quad \text { case B. }
\end{aligned}
$$

Using the recurrence relation Eq. (A1) and the offspring generating function Eq. (B3), one can obtain [34] the general form of $G^{(s)}\left(x \mid \gamma_{s}\right)$ as

$$
G^{(s)}\left(x \mid \gamma_{s}\right)=\frac{a_{s}+b_{s} x}{c_{s}+d_{s} x},
$$

where the values of the constants $a_{s}, b_{s}, c_{s}$, and $d_{s}$ satisfy [for a log-balanced noise (case $\mathrm{A})]$ the recurrence relation

$$
\begin{aligned}
& {\left[\begin{array}{l}
a_{s+1} \\
c_{s+1} \\
b_{s+1} \\
d_{s+1}
\end{array}\right]} \\
& =\left[\begin{array}{cccc}
0 & 1 & 0 & 0 \\
-e^{\gamma_{s+1}} & e^{\gamma_{s+1}}+1 & 0 & 0 \\
0 & 0 & 0 & 1 \\
0 & 0 & -e^{\gamma_{s+1}} & e^{\gamma_{s+1}}+1
\end{array}\right]\left[\begin{array}{l}
a_{s} \\
c_{s} \\
b_{s} \\
d_{s}
\end{array}\right]
\end{aligned}
$$

with the initial conditions $a_{1}=1, b_{1}=0, c_{1}=e^{\gamma_{1}}+1$ and $d_{1}=-e^{\gamma_{1}}$. The corresponding equations in case $\mathrm{B}$ are obtained by replacing $e^{\gamma}$ by $1+\gamma$ in all the above expressions.

The chance of survival until the $s$ th generation $\delta_{s} \equiv 1-$ $G^{s}(0)$ is simply

$$
\delta_{s}=1-a_{s} / c_{s} .
$$

Accordingly, the survival probability satisfies the stochastic recursion relations:

$$
\begin{aligned}
\delta_{s+1} & =\frac{\delta_{s}}{e^{-\gamma_{s}}+\delta_{s}}, \quad \text { case } \mathrm{A}, \\
\delta_{s+1} & =\frac{(1+\gamma) \delta_{s}}{1+(1+\gamma) \delta_{s}}, \quad \text { case B. }
\end{aligned}
$$

When $\delta_{s} \ll 1$, it satisfies $\delta_{s+1}=\delta_{s} e^{\gamma_{s}}-\delta_{s}^{2}$ in case A and $\delta_{s+1}=(1+\gamma) \delta_{s}-\delta_{s}^{2}$ in case B. One has to be careful in translating the equation for case $\mathrm{A}$ to a stochastic differential equation, as $\exp \left(\gamma_{s}\right)$ has a nonzero expectation value, namely $\cosh \sqrt{2 D}$. Thus, in terms of $\delta$ there is an extra term driving $\delta$ to larger values. Taking this into account, in the weak environmental noise limit, $\gamma_{s} \ll 1$, one gets the stochastic differential equations that were quoted in the main text:

$$
\begin{aligned}
& \frac{d \delta(t)}{d t}=\gamma_{t} \delta(t)+D \delta(t)-\delta^{2}(t), \quad \text { case A, } \\
& \frac{d \delta(t)}{d t}=\gamma_{t} \delta(t)-\delta^{2}(t), \quad \text { case B. }
\end{aligned}
$$

\section{Long-time asymptotics}

The Langevin equations (B7) should be interpreted in the Ito sense, since they were derived as the white noise, continuous time limit of a nonoverlapping generation model that assumes zero relaxation time, i.e., that the population follows the instantaneous growth rate determined by $\gamma[22,23]$. When the relaxation time of the population or the community is finite (i.e., when the demographic rates respond slowly to the changing environment, with respect to the noise correlation time) the effective strength of the environmental noise amplitude decreases, but the interpretation is still Ito. Accordingly, in the logarithmic space $y=\ln (\delta)$, Eqs. (B7) takes the form

$$
\begin{aligned}
& \frac{d y}{d s}=\gamma(s)-\exp (y), \quad \text { case A, } \\
& \frac{d y}{d s}=\gamma(s)-D-\exp (y), \quad \text { case B. }
\end{aligned}
$$

It is clear, now, that as $\delta \rightarrow 0$, i.e., $y \rightarrow-\infty$, in case $\mathrm{A}$ the system performs an unbiased random walk in the log space, 


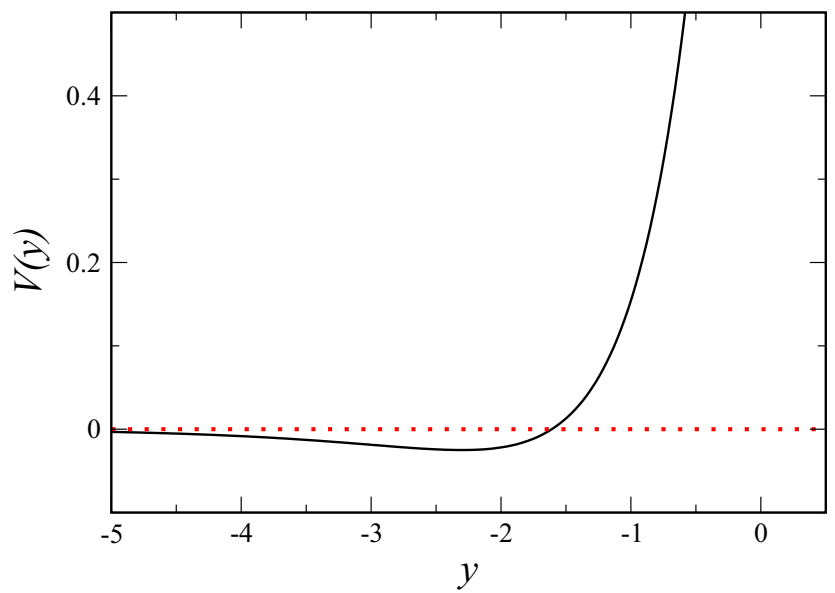

FIG. 5. (Color online) The Morse potential $V(y)=$ $\exp (2 y) / 4 D-\exp (y) / 2$ for $D=0.1$.

hence in the long run one expects that the probability of a taxon to have a lifetime $t$ will behave like $t^{-3 / 2}$, i.e., that $\delta(t) \sim t^{-1 / 2}$. In case $\mathrm{B}$, on the other hand, the random walk is biased to the left, and the survival probability should decrease exponentially in time.

To demonstrate that, we notice that the corresponding Fokker-Planck equations are

$$
\begin{aligned}
& \partial_{s} P(y, s)=D \partial_{y}^{2} P(y, s)+\partial_{y}[\exp (y) P(y, s)], \quad \text { case A, } \\
& \partial_{s} P(y, s)=D \partial_{y}^{2} P(y, s)+\partial_{y}\{[D+\exp (y)] P(y, s)\}, \quad \text { case B. }
\end{aligned}
$$

With the substitutions $P(y, s)=\exp \left(-e^{y} / 2 D\right) \psi(y, s)$ (A) and $P(y, s)=\exp \left(-y / 2-e^{y} / 2 D\right) \psi(y, s)(\mathrm{B})$ one gets

$$
\begin{aligned}
& \dot{\psi}(y, s)=D \psi^{\prime \prime}+\left(\frac{\exp (y)}{2}-\frac{\exp (2 y)}{4 D}\right) \psi, \quad \text { case A, } \\
& \dot{\psi}(y, s)=D \psi^{\prime \prime}-\left(\frac{\exp (2 y)}{4 D}+\frac{D}{4}\right) \psi, \quad \text { case B. }
\end{aligned}
$$

These are Schrodinger equations in imaginary time with an exponentially decaying potential (case B) and a Morse potential (case A; see Fig. 5).

A wave packet which is initially localized at small negative values of $y$ (corresponding to $\delta \sim 1$ ) will eventually reach the region of large negative $y(\delta \rightarrow 0)$, where the potential is negligible and the motion is almost purely diffusive. This is clearer in case $\mathrm{B}$, where the long-term behavior of $\psi$ (neglecting the exponential term) is

$$
\psi(y, t) \approx \frac{e^{-y^{2} / 4 D t} e^{-D t / 4}}{t}
$$

so the wave function diffuses in the log space, but this diffusion is superimposed of an exponential decay exp $(-D t / 4)$ (we have replaced the generation parameter $s$ by time $t$, as in the long term the changes of $\delta$ over a single generation are small). For case A an asymptotic matching approach leads to the uniform approximation

$$
P(\delta, t) \approx \frac{1}{\delta \sqrt{\pi D t}} e^{-\left[\ln (\delta / D)+\gamma_{E}\right]^{2} / 4 D t} e^{-\delta / D},
$$

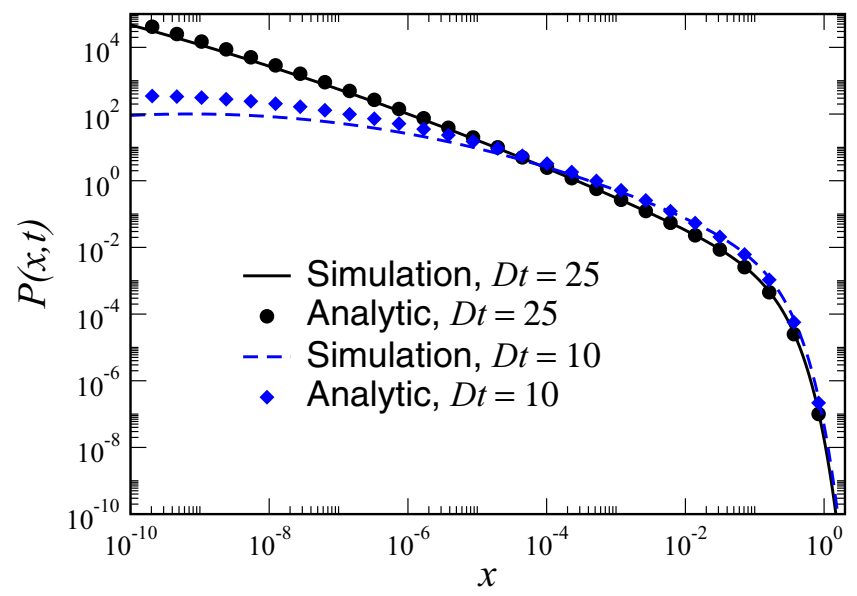

FIG. 6. (Color online) Numerical simulation of the FokkerPlanck equation (B7), compared to the analytic approximation, Eq. (B12), for $D=0.1, D t=10$, and $D t=25$.

where $\gamma_{E}$ is the Euler constant. This approximate solution is shown in Fig. 6, together with a direct numerical solution of the Fokker-Planck equation (B7). We see that the agreement is very good, and improves with time. At long times, the expectation value of $\delta$ is $\bar{\delta} \approx \sqrt{D / \pi}$, so that the extinction rate has a $t^{-3 / 2}$ tail. For case $\mathrm{B}$ we have

$$
P(\delta, t) \approx \frac{1}{\delta \sqrt{\pi D t}} e^{(\ln \delta+D) / 4 D t}
$$

so that the log-normal distribution moves toward negative values of $\ln (\delta)$ at a constant rate, leading to a typical $\delta$ which decays exponentially in time.

\section{Abundance-age relationship}

We now turn to analyze the effect of environmental noise on the abundance-age relationship in neutral theory. As explained in the main text, there are two ingredients that determine the age-abundance relationships: the chance of a species to reach abundance $N$ after $s$ generations, and its chance of survival. The ratio between these factors gives $N_{\text {surv }}$, the average size of a surviving species. The chance of survival was calculated above, and now we will calculate the average abundance.

In case B the result is quite trivial: since the demographic and the environmental stochasticity are both balanced in the abundance space, the average abundance of a species is fixed. Accordingly, we perform the analysis here for case A, and will explain at the end how the results relate to our prediction for case B.

From Eq. (B4), we can see that the width of the geometric distribution of the abundance at time $s$ is given by $\zeta(s)=$ $-1 / \ln \left(-d_{s} / c_{s}\right)$. Note that $d_{s}$ and $c_{s}$ satisfy the same recurrence relations, and the only difference between them is the initial conditions. This allows us to consider only $d_{s}$ and then to carry over the results to $c_{s}$ and hence calculate their ratio. From (B5) it is clear that $d_{s}$ satisfies

$$
d_{s+1}-d_{s}=e^{\gamma_{s}}\left(d_{s}-d_{s-1}\right)
$$


The solution to this is easily verified to be

$$
d_{s}=d_{1} \sum_{k=1}^{s} \prod_{j=2}^{k} e^{\gamma_{j}} .
$$

Given $d_{1}=-e^{\gamma_{1}}$, we can write this as

$$
d_{s}=-\sum_{k=1}^{s} \prod_{j=1}^{k} e^{\gamma_{j}} \text {. }
$$

As explained above, the equations for $c$ is the same, except that $c_{1}=1+e^{\gamma_{1}}$, so that

$$
c_{s}=\sum_{k=0}^{s} \prod_{j=1}^{k} e^{\gamma_{j}} .
$$

Thus, $d_{s}=-\left(c_{s}-1\right)$ and so $1 / \zeta=-\ln \left(-d_{s} / c_{s}\right)=-\ln (1-$ $\left.1 / c_{s}\right)$. It should first be noted that for $s \gg 1, D \ll 1, c_{s}$ is large. For the case of binary noise, for example, $\gamma= \pm \gamma_{0}$, the minimum possible value of $c_{s}$ is for the case where $\gamma_{s}$ is always negative, in which case $c_{s} \approx 1 / \gamma_{0}$. Thus, $\zeta \approx c_{s}$, so that

$$
\bar{\zeta} \approx \sum_{k=0}^{s} \overline{\prod_{j=1}^{k} e^{\gamma_{j}}}=\sum_{k=0}^{s} e^{D k / 2}=\frac{2}{D}\left(e^{D s / 2}-1\right) .
$$

So we see that $\overline{c_{s}}$ is exponentially large for large $s$ (see Fig. 7).

For case $\mathrm{B}$, as explained, one should replace $e^{\gamma}$ by $1+\gamma$, and the trivial result is a linear growth, $\zeta \sim t$.

The result (B18) has two limits: when $s \ll 1 / D$, the environmental noise is negligible and the average abundance of a species (conditioned on its presence in the community) grows linearly in time. However, at long times the environmental noise controls the system and the typical size of surviving species grows exponentially with $s$. In this regime the typical time scale (in generations) needed for a species to reach abundance $N$ will scale like $\ln (N) / D$, as opposed to the linear $N$ scaling for pure demographic noise.

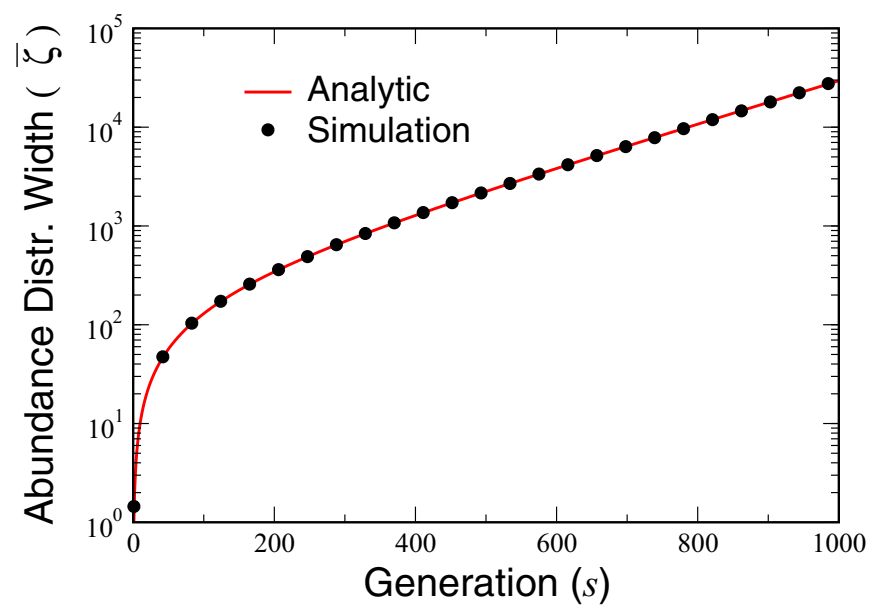

FIG. 7. (Color online) A graph of the ensemble-averaged width $\overline{\zeta(s)}$ calculated by averaging $\zeta$ as computed from the analytic generating function Eq. (B5) over $10^{6}$ realizations of binary noise $\gamma=$ $\pm \gamma_{0}$, with $\gamma_{0}=0.1$. Also shown is the analytic formula, Eq. (B18).

\section{Average vs typical age-abundance relationships in case $A$}

Our result for case A, Eq. (B18), might appear at first glance to be in contradiction to the results proved by Geiger and collaborators [35]. These authors show that, in case A,

$$
\lim _{s \rightarrow \infty} \frac{\ln \left[E\left(N_{\text {surv }}\right)\right]}{s}=0,
$$

and decided that this log-balanced scenario is "critical," as opposed to case B (or, in general, any noise which gives a negative bias in the log space) which is subcritical.

To explain this apparent contradiction, let us stick to the method used in Ref. [35], averaging first over demographic noise. In the log-abundance space, what one has after taking this average is a random walker that starts at zero (one individual) and moves randomly with no bias until $s$. Clearly, the population at $s$, conditioned on survival and averaged over demographic noise, is $\exp (y)$, where $y$ is the location of a random walker that starts at $s=0$ and subsequently never crosses zero, since otherwise it goes extinct. Such a constrained random walk (RW) is known as a meander. To calculate the probability that the meander is at $y$ at time $s$ one solves for a RW that starts at $x_{o}$ using images, taking $x_{0}$ to zero at the end. The result is

$$
P(y, s)=A \frac{y e^{-y^{2} / 4 D s}}{s^{3 / 2}} .
$$

Accordingly, the maximum likelihood for the position of the meander at $s$ is (for large $s$ ),

$$
Y_{M L}=\sqrt{2 D s} .
$$

This implies that the typical size of a population at $s$, conditioned on nonextinction, is

$$
N_{\text {surv }}^{\text {typ }} \approx e^{y}=\exp (\sqrt{2 D s}) .
$$

On the other hand, the average value of $N_{\text {surv }}$ will be

$$
N_{\text {surv }} \approx \int d y e^{y} \frac{y e^{-y^{2} / 4 D s}}{s^{3 / 2}} \approx e^{D t} .
$$

Note that the result for the average comes from the peak of the integrand at $y^{*}=2 D s$ (this is a Laplace integral) and $P\left(y^{*}\right)$ is exponentially small as $s \rightarrow \infty$, so this result is consistent with [35] since the average comes from exponentially rare events (with probability 1 this will not be the case for any specific history). On the other hand, $P\left(y_{M L}\right)$ is $\mathcal{O}(1 / t)$, which is not negligible. However, the typical growth is subexponential, and clearly agrees with (B19).

\section{APPENDIX C: UNIVERSALITY}

Throughout Appendix B we have studied the geometric neutral process because of its convenient properties. Of course, any result that depends on the specific properties of a certain distribution cannot be relevant to the generic case, where the distribution of number of descendants per individual is, in most cases, unknown and there is no reason to believe that it belongs to any particular simple distribution. 
To illustrate the generality of our results, let us consider the survival probability $\delta_{s}$. In the generic case one may define a probability distribution function $P_{n}(s)$, the chance of an individual to produce $n$ offspring during the $s$ generation, and of course the two important summary statistics that characterize this distribution are its mean [in case A, $\bar{n}=\exp \left(\gamma_{s}\right)$, in case $\left.\mathrm{B}, \bar{n}=\gamma_{s}\right]$ and its variance. Here we assume that the environmental noise is weak so one can neglect the variance fluctuations and $\operatorname{var}(n)=\sigma^{2}$, where $\sigma^{2}$ is the variance of the purely demographic model $\left(\sigma^{2}=1\right.$ for Poisson distribution with average $1, \sigma^{2}=2$ for the geometric distribution, and so on).

The generating function recursion relation implies that

$$
G^{(s)}(x)=G_{s}^{(1)}\left(G^{(s-1)}(x)\right) .
$$

In the long-time limit the chance to survive is small, so $G^{(s)}(x)=1-\delta_{s}$. Plugging this into (C1) one gets

$$
\begin{aligned}
1-\delta_{s} & =\sum_{n} P_{n}(s)\left(1-\delta_{s-1}\right)^{n} \\
& \approx \sum_{n} P_{n}(s)\left(1-n \delta_{s-1}+\frac{n^{2}-n}{2} \delta_{s-1}^{2}\right) .
\end{aligned}
$$

Accordingly,

$$
\begin{aligned}
& \dot{\delta} \approx \delta_{s}-\delta_{s-1}=\left(e^{\gamma_{s}}-1\right) \delta-\frac{\sigma^{2}}{2} \delta^{2}, \quad \text { case A, } \\
& \dot{\delta}=\gamma_{s} \delta-\frac{\sigma^{2}}{2} \delta^{2}, \quad \text { case B. }
\end{aligned}
$$

The second equation is what appears in Eq. (4) of the main text. In case A one needs to expand the exponent in $\gamma$ and to replace $\gamma^{2} / 2$ by $D$ to obtain the equation presented in the main text.
[1] M. Kimura, The Neutral Theory of Molecular Evolution (Cambridge University Press, Cambridge, England, 1984).

[2] S. P. Hubbell, The Unified Neutral Theory of Biodiversity and Biogeography, Monographs in Population Biology 32 (Princeton University Press, Princeton, NJ, 2001).

[3] R. Muneepeerakul, E. Bertuzzo, H. J. Lynch, W. F. Fagan, A. Rinaldo, and I. Rodriguez-Iturbe, Nature (London) 453, 220 (2008).

[4] J. Rosindell, S. P. Hubbell, and R. S. Etienne, Trends Ecol. Evol. 26, 340 (2011).

[5] I. Volkov, J. R. Banavar, S. P. Hubbell, and A. Maritan, Nature (London) 424, 1035 (2003).

[6] P. A. Marquet, A. P. Allen, J. H. Brown, J. A. Dunne, B. J. Enquist, J. F. Gillooly, P. A. Gowaty, J. L. Green, J. Harte, S. P. Hubbell et al., BioScience 64, 701 (2014).

[7] S. R. Connolly, M. A. MacNeil, M. J. Caley, N. Knowlton, E. Cripps, M. Hisano, L. M. Thibaut, B. D. Bhattacharya, L. Benedetti-Cecchi, R. E. Brainard et al., Proc. Natl. Acad. Sci. USA 111, 8524 (2014).

[8] S. Nee, Funct. Ecol. 19, 173 (2005).

[9] R. E. Ricklefs, Ecology 87, 1424 (2006).

[10] A. P. Allen and V. M. Savage, Ecol. Lett. 10, 637 (2007).

[11] R. A. Chisholm and J. P. O'Dwyer, Theor. Pop. Biol. 93, 85 (2014).

[12] J. Rosindell, S. J. Cornell, S. P. Hubbell, and R. S. Etienne, Ecol. Lett. 13, 716 (2010).

[13] E. G. Leigh, J. Evol. Biol. 20, 2075 (2007).

[14] K. J. Feeley, S. J. Davies, R. Perez, S. P. Hubbell, and R. B. Foster, Ecology 92, 871 (2011).

[15] M. Kalyuzhny, E. Seri, R. Chocron, C. H. Flather, R. Kadmon, and N. M. Shnerb, Am. Nat. 184, 439 (2014).

[16] R. A. Chisholm, R. Condit, K. A. Rahman, P. J. Baker, S. Bunyavejchewin, Y.-Y. Chen, G. Chuyong, H. S. Dattaraja, S. Davies, C. E. N. Ewango et al., Ecol. Lett. 17, 855 (2014).
[17] M. Kalyuzhny, Y. Schreiber, R. Chocron, C. H. Flather, R. Kadmon, D. A. Kessler, and N. M. Shnerb, Ecology 95, 1701 (2014).

[18] M. Kalyuzhny, R. Kadmon, and N. M. Shnerb, Ecol. Lett. 18, 572 (2015).

[19] A. E. Magurran and P. A. Henderson, Nature (London) 422, 714 (2003).

[20] P. B. Adler, W. R. Tyburczy, and W. K. Lauenroth, Ecology 88, 2673 (2007).

[21] R. C. Lewontin and D. Cohen, Proc. Natl. Acad. Sci. USA 62, 1056 (1969).

[22] R. Kupferman, G. A. Pavliotis, and A. M. Stuart, Phys. Rev. E 70, 036120 (2004).

[23] S. Suweis, A. Porporato, A. Rinaldo, and A. Maritan, Phys. Rev. E 83, 061119 (2011).

[24] S. Pigolotti, A. Flammini, M. Marsili, and A. Maritan, Proc. Natl. Acad. Sci. USA 102, 15747 (2005).

[25] E. Bertuzzo, S. Suweis, L. Mari, A. Maritan, I. Rodríguez-Iturbe, and A. Rinaldo, Proc. Natl. Acad. Sci. USA 108, 4346 (2011).

[26] S. Suweis, E. Bertuzzo, L. Mari, I. Rodriguez-Iturbe, A. Maritan, and A. Rinaldo, J. Theor. Biol. 303, 15 (2012).

[27] F. Galton and H. W. Watson, J. R. Anthropol. Inst. 4, 138 (1874).

[28] D. A. Kessler and N. M. Shnerb, J. Theor. Biol. 345, 1 (2014).

[29] J. Geiger and G. Kersting, Theor. Probab. Appl. 45, 517 (2001).

[30] S. Azaele, S. Pigolotti, J. R. Banavar, and A. Maritan, Nature (London) 444, 926 (2006).

[31] D. R. Hekstra and S. Leibler, Cell 149, 1164 (2012).

[32] M. Loreau and C. de Mazancourt, Am. Nat. 172, E48 (2008).

[33] H. ter Steege, N. C. Pitman, D. Sabatier, C. Baraloto, R. P. Salomão, J. E. Guevara, O. L. Phillips, C. V. Castilho, W. E. Magnusson, J.-F. Molino et al., Science 342, 1243092 (2013).

[34] J. F. Steffensen, Annales de l'Inst. Henri Poincaré 3, 319 (1933).

[35] J. Geiger, G. Kersting, and V. A. Vatutin, Annales de l'Institut Henri Poincare (B) 39, 593 (2003). 